\title{
Exome sequencing: value is in the eye of the beholder
}

\author{
Scott D. Grosse, PhD ${ }^{1}{ }^{1}$ and Sonja A. Rasmussen, MD, MS (id ${ }^{2,3}$ \\ Genetics in Medicine (2020) 22:280-282; https://doi.org/10.1038/s41436-019-0674-Z
}

Recent studies have discussed the benefits of using exome sequencing (ES) as part of the genetics evaluation of early onset seizure disorders, ${ }^{1}$ neurodevelopmental disorders, ${ }^{2}$ and acute illness in newborns of suspected genetic origin, ${ }^{3}$ among others. However, barriers to the clinical use of ES include a widespread reluctance of insurers to pay for testing. ${ }^{4}$ This leaves researchers who develop and evaluate genetic tests and clinicians who must make the case for their patient's need for genetic testing with questions regarding what evidence would be required to ensure coverage and patient access.

Coverage of ES by US health-care payers has until recently been uncommon. An important source of information on payer coverage of genetic testing is the University of California-San Francisco (UCSF) Center for Translational and Policy Research on Personalized Medicine (TRANSPERS) Payer Coverage Policy Registry@. A 2015 survey found that a minority of plans covered multigene tests of any kind and none covered ES. ${ }^{5}$ During 2014-2017, multigene panels accounted for roughly $60 \%$ of private plan spending on genetic testing and $<2 \%$ was spent on exome or genome sequencing. ${ }^{6}$ Two recent analyses from the UCSF Program in Prenatal and Pediatric Genomic Sequencing (P3EGS) have reported that growing numbers of health plans are now covering pediatric ES for at least some indications. One study reported that 8 of 15 payers surveyed in 2017 covered ES for children. ${ }^{7}$ In this issue, Trosman et al. report on qualitative research on factors underlying those evolving coverage decisions. ${ }^{8}$

What drives payers' coverage decisions for genetic testing is not well understood. Payers often cite the lack of data on "clinical utility" as justification for noncoverage, ${ }^{9}$ but the understanding of what constitutes clinical utility varies. Does clinical utility refer specifically to improved health outcomes or does it also embrace the ability of a molecular genetic diagnosis to guide treatment options and to end diagnostic odysseys? Clinical utility has often been defined by researchers and health policy experts in terms of net health outcomes (death and serious disease or disability) ${ }^{10}$ whereas clinical geneticists, including the American College of Medical
Genetics and Genomics (ACMG), have defined clinical utility more broadly to include effects on clinical management, prognostic implications, benefits of the information for patients and their family members, and the economic impact on health-care systems. ${ }^{11}$ Others have proposed that the personal utility of genomic information be considered independent of its use to alter clinical management or outcomes. ${ }^{12}$ Finally, interpretation of evidence differs; Douglas et al. reported that the same clinical utility studies were cited by some plans as reasons to cover pediatric ES and by other plans as grounds for negative decisions. ${ }^{7}$

TRANSPERS investigators have previously investigated reasons for coverage decisions for other types of genetic testing. For example, Dervan et al. reported that as of early 2016 just 8 of 19 payers covered cell-free fetal DNA testing for aneuploidies in average-risk pregnancies. ${ }^{13}$ Most (15/19) plans cited evidence of clinical validity in their decisionmaking process, and the majority (11/19) also cited clinical utility, which was defined in all policies as the expected change in net health outcomes. Three of the plans also considered change in patient management or clinical decision-making as part of the definition of clinical utility.

Similarly, TRANSPERS surveys of representatives of payers regarding why multigene panels of tumor testing for hereditary cancers were not covered found that the primary reason offered was that such testing does not fit the standard coverage framework that requires evidence of clinical benefit to justify a treatment as "medically necessary." 14,15 That is, although there might be sufficient evidence of clinical benefit from identification of some gene variants, because other variants with unclear implications are also identified, the test as a whole would be classified as investigational. In addition, it is reported that payers rely on clinical guidelines in coverage decisions on genetic testing to a larger extent than for drugs. ${ }^{16}$ Also, it was reported that evidence of cost-effectiveness was rarely considered, and budget impact, i.e., net cost savings, was not cited in any of the coverage decisions reviewed. ${ }^{16}$

In this issue, Trosman et al. provide data to help understand payer coverage decisions for pediatric and prenatal ES. ${ }^{8}$ They

\footnotetext{
${ }^{1}$ National Center on Birth Defects and Developmental Disabilities, Centers for Disease Control and Prevention, Atlanta, GA, USA; ${ }^{2}$ Department of Pediatrics, University of Florida College of Medicine, Gainesville, FL, USA; ${ }^{3}$ Department of Epidemiology, University of Florida College of Medicine and College of Public Health and Health Professions, Gainesville, FL, USA. Correspondence: Sonja A. Rasmussen (skr9@ufl.edu)
}

Submitted 16 September 2019; accepted: 27 September 2019

Published online: 14 October 2019 
interviewed representatives of 14 payers and found that some payers' views of factors needed to approve coverage were expanding to include informational utility and ending the diagnostic odyssey. Ten of 14 (71\%) payer representatives noted that their organizations approved coverage for pediatric ES, while none approved coverage of ES in the prenatal period. Although the payer representatives noted that they felt the evidence for clinical utility (better net health outcomes) of pediatric ES was insufficient, they recognized the benefits of the information that ES provided $(70 \%)$ or of ending the diagnostic odyssey faced by families (30\%). Representatives of five payers that reported covering pediatric ES expressed concerns about potentially inappropriate use or expanding indications and difficulty interpreting test results. Consequently, many payers have set limits for which clinical scenarios would have ES reimbursed or which specialties could order ES. Also, some plans have implemented prior authorization and utilization management programs. Half of payers acknowledged the informational utility of ES and three considered it sufficient for coverage, but most saw it as secondary to clinical utility. Most payers considered the ability to end diagnostic odysseys an important aspect of clinical utility; several considered that to be sufficient grounds for coverage. In contrast, payers did not see any advantage of prenatal ES over prenatal ultrasound and array-based genetic testing, given the sparse evidence base for prenatal ES as well as the absence of diagnostic odysseys.

Decisions as to which genetic tests are reimbursed by thirdparty payers may be shaped as much by payers' perceptions and preferences as by evidence regarding benefits and costs, which is reflected in inconsistency in payer coverage decisions. The perceived "value" of genetic tests, i.e., the expected net benefit relative to expected costs, depends in large part on which benefits are considered to matter. As with beauty, value is in the eye of the beholder. Health-care payers have traditionally focused on "hard" measures of health outcomes. Some have proposed that outcomes that matter to patients, i.e., personal utility, also be considered. Ending the diagnostic odyssey involves both personal and informational utility, the value of knowing, as well as clinical utility in terms of treatment options.

Until now, US payers seem to have been reluctant to consider personal utility in coverage decisions on genetic testing. The findings reported by Trosman and others in this issue represent the first concrete evidence that US third-party payers are beginning to consider the ACMG-recommended definition of clinical utility as justification for paying for genetic testing independent of evidence of improved health outcomes. One possible explanation for the willingness of payers to cover genetic tests that may not satisfy traditional criteria for coverage decisions, besides falling costs, is the recognition that consumer satisfaction may influence commercial health plan market shares.

Because coverage by payers is necessary for advances in genomic medicine to be made accessible to patients, a better understanding of how payers value genetic testing applications is critical. We applaud Trosman and colleagues for working to address what matters to payers in making coverage decisions for ES. It is encouraging to see that payer definitions of the value of genetic testing are expanding, at least for pediatric ES, and in some cases appear to be approaching that of parents and clinicians. However, although this study represented the views of 14 payers who collectively cover more than 170 million enrollees, it is not known how representative these payers are of all payers. In addition, the study did not evaluate payers' views of the value of other types of genetic testing, such as multigene testing for hereditary cancers or pre-emptive pharmacogenetic testing. As advances in genetic testing increase, additional research into how payer coverage decisions are made could help to ensure that these advances benefit the patients for whom they were developed.

Coverage by payers of new technologies, such as ES, is not necessarily a binary process. Even if a plan agrees to pay for ES, restrictions on which groups of patients are considered eligible for ES, which providers are allowed to order ES and be reimbursed, or the various bureaucratic hoops and hurdles that providers may be required to surmount to obtain agreement by payers to reimburse for ES might deter some providers and patients from obtaining ES. In addition, high copays or deductibles can deter patients from following through on recommended treatments, resulting in suboptimal uptake. Many patients with undiagnosed disease who are able to benefit from ES provided in a research setting are reported to have previously not been sequenced because of perceived barriers to insurance coverage, ${ }^{4}$ but the specific types of barriers were not identified. Studies of payer perspectives of the coverage process for pediatric ES, such as that of Trosman and others, could be usefully complemented by surveys of provider and patient perspectives on barriers to effective access to reimbursement for ES.

\section{DISCLOSURE}

The authors declare no conflicts of interest.

\section{DISCLAIMER}

The findings and conclusions in this report are those of the authors and do not necessarily represent the official position of the Centers for Disease Control and Prevention.

Publisher's note Springer Nature remains neutral with regard to jurisdictional claims in published maps and institutional affiliations.

\section{REFERENCES}

1. Berg AT, Coryell J, Saneto RP, et al. Early-life epilepsies and the emerging role of genetic testing. JAMA Pediatr. 2017;171:863-871.

2. Srivastava S, Love-Nichols JA, Dies KA, et al. Meta-analysis and multidisciplinary consensus statement: exome sequencing is a first-tier clinical diagnostic test for individuals with neurodevelopmental disorders. Genet Med. 2019. https://doi.org/10.1038/s41436-019-0554-6 [Epub ahead of print].

3. Friedman JM, Bombard Y, Cornel MC, et al. Genome-wide sequencing in acutely ill infants: genomic medicine's critical application? Genet Med. 2019;21:498-504. 
4. Reuter CM, Kohler JN, Bonner D, et al. Yield of whole exome sequencing in undiagnosed patients facing insurance coverage barriers to genetic testing. J Genet Couns. 2019. https://doi.org/10.1002/jgc4.1161 [Epub ahead of print].

5. Phillips KA, Deverka PA, Trosman JR, et al. Payer coverage policies for multigene tests. Nat Biotechnol. 2017;35:614-617.

6. Phillips KA, Deverka PA, Hooker GW, Douglas MP. Genetic test availability and spending: Where are we now? Where are we going? Health Aff (Millwood). 2018;37:710-716.

7. Douglas MP, Parker SL, Trosman JR, et al. Private payer coverage policies for exome sequencing (ES) in pediatric patients: trends over time and analysis of evidence cited. Genet Med. 2019;21:152-160.

8. Trosman JR, Weldon CB, Slavotinek AM, et al. Perspectives of US private payers on insurance coverage for pediatric and prenatal exome sequencing: results of a study from the Program in Prenatal and Pediatric Genomic Sequencing (P3EGS). Genet Med. 2019. https://doi org/10.1038/s41436-019-0650-7 [Epub ahead of print].

9. Pezalla EJ. Payer view of personalized medicine. Am J Health Syst Pharm. 2016:73:2007-2012.
10. Grosse SD, Khoury MJ. What is the clinical utility of genetic testing? Genet Med. 2006;8:448-450.

11. ACMG Board of Directors. Clinical utility of genetic and genomic services: a position statement of the American College of Medical Genetics and Genomics. Genet Med. 2015;17:505-507.

12. Foster MW, Mulvihill JJ, Sharp RR. Evaluating the utility of personal genomic information. Genet Med. 2009;11:570-574.

13. Dervan AP, Deverka PA, Trosman JR, et al. Payer decision making for next-generation sequencing-based genetic tests: insights from cell-free DNA prenatal screening. Genet Med. 2017;19:559-567.

14. Trosman JR, Weldon CB, Kelley RK, Phillips KA. Challenges of coverage policy development for next-generation tumor sequencing panels: experts and payers weigh in. J Natl Compr Canc Netw. 2015;13:311-318.

15. Trosman JR, Weldon CB, Douglas MP, et al. Payer coverage for hereditary cancer panels: barriers, opportunities, and implications for the precision medicine initiative. J Natl Compr Canc Netw. 2017;15:219-228.

16. Chambers JD, Saret CJ, Anderson JE, et al. Examining evidence in U.S. payer coverage policies for multi-gene panels and sequencing tests. Int J Technol Assess Health Care. 2017;33:534-540. 